\title{
Sistema de gestión de seguridad y salud en el trabajo, para la empresa de vialidad IMBAVIAL E.P. Provincia de Imbabura
}

\section{RESUMEN}

El presente trabajo de investigación fue llevado a cabo en la empresa INBAVIAL E.P., cuya actividad económica es de ingeniería civil el riesgo es alto acorde a lo que establece la categorización. El propósito de este tema de investigación es implementar un sistema de Gestión de Seguridad y Salud Ocupacional basado en la legislación Ecuatoriana, este sistema de gestión permite tener un conocimiento a nivel administrativo, técnico, talento humano y operativo, esto quiere decir que son cuatro pilares fundamentales los que ejecuta el sistema de gestión, para ello se parte de un manual, el mismo que tiene procedimientos e instructivos, formatos para registros los cuales son auditables y verificables, que permiten cumplir con el ciclo de Deming.

Al ejecutar este tema de investigación se comprueba que el sistema de gestión de seguridad y salud ocupacional mejora las condiciones de trabajo de la empresa, debido a que se tiene un control total de cada una de las actividades y condiciones de trabajo. El cambio de esta empresa es radical debido a que la gerencia apoya con los recursos económicos, tecnológicos y humanos buscando la prevención de riesgos laborales (PRL), promoviendo la seguridad y salud de los trabajadores mediante la identificación, evaluación y control de los peligros y riesgos asociados a un entorno laboral, además de fomentar el desarrollo de actividades y medidas necesarias para prevenir los riesgos.

Palabras clave: Sistema de gestión de calidad, minimizar riesgos, ciclo de deming, prevención de riesgos.

OCCUPATIONAL HEALTH AND SAFETY MANAGEMENT SYSTEM FOR THE MOTORWAY COMPANY IMBAVIAL E.P. Province OF IMBabURA

ABSTRACT

The present research work was carried out in the company INBAVIAL E.P., whose economic activity is of civil engineering the risk is high according to what the categorization establishes. The purpose of this research topic is to implement a system of Occupational Health and Safety Management based on Ecuadorian legislation, this management system allows to have an administrative, technical, human and operational talent, which means that they are four pillars Which are executed by the management system, for it is part of a manual, which has procedures and instructions, formats for records that are auditable and verifiable, that allow to comply with the Deming cycle. In carrying out this research, it is verified that the occupational health and safety management system improves the working conditions of the company, due to the total control of each of the activities and working conditions. The change of this company is radical because the management supports with the economic, technological and human resources looking for the prevention of occupational risks (PRL) promoting the safety and health of the workers through the identification, evaluation and control of the dangers and Risks associated with a work environment, in addition to encouraging the development of activities and measures necessary to prevent risks.

Keywords: Quality management system, minimize risks, deming cycle, risk prevention.

\section{INTRODUCCIÓN}

Durante el período 2014, la Dirección General del Seguro de Riesgos del Trabajo, desplegó un control permanente con el proceso de auto-auditorías en las cuales participaron más de 15500 empresas y posterior a esto, se desarrolló el proceso de auditorías presenciales con 122 empresas, a través de la prevención y control se reducirán gastos en reparación y compensaciones. En cuanto a atención médica, existen 3496 casos en enfermedades profesionales y 37183 en accidentes de trabajo, con el $75 \%$ de atenciones en Guayas y Pichincha. La siniestralidad reportada corresponde el $97 \%$ a accidentes de trabajo y el $3 \%$ a enfermedades profesionales, (Vazques, 2014)

Luis Vazques muestra el funcionamiento del sistema de control que desarrolló la Dirección de Riesgos del Trabajo del Instituto Ecuatoriano Seguridad Social e indica que por cada 1000 trabajadores 42 trabajadores sufren accidentes. Cada día, 6400 personas fallecen debido a un accidente del trabajo o a una enfermedad profesional, y las muertes por esta causa ascienden a 2,3 millones anuales. "Sin duda, los accidentes del trabajo y las enfermedades profesionales son una de las principales cargas para los sistemas de salud en el mundo" (Torres, 2015, pág. 1).

Considerando lo que establece el Ing. Vásquez la Empresa de Vialidad IMBAVIAL en el mes de mayo del 2014 incumplió la legislación vigente a nivel nacional, no declarando un accidente laboral, trayendo consecuencias a la empresa con una glosa de un salario básico, es por todo aquello que se ha ido implementando el sistema de gestión de seguridad y salud en el trabajo con el objetivo de mantener un ambiente de trabajo adecuado minimizando los accidentes y enfermedades laborales en la empresa. El sistema de gestión de seguridad y salud en el trabajo mantiene 4 aspectos elementales que son: gestión administrativa, gestión talento humano, gestión técnica y procesos y programas operativos básicos, (Jaramillo, 2011).

1 Magister en Diseño, Gestión y Evaluación de Proyectos Sociales y Productivos, Doctor en Ingeniería Industrial, Docente Investigador Titular de la Facultad de Ingeniería de la Universidad Nacional de Chimborazo, Riobamba, Ecuador.

E-mail: mcabrera@unach.edu.ec

2 Magister en seguridad industrial mención prevención de riesgos y salud ocupacional, Jefe del departamento de Seguridad y salud ocupacional de INBAVIAL.

E-mail: guvidia@imbavial.gob.ec

3 Magíster en Gestión de la Producción, Doctor en Ingeniería Industrial, Docente Investigador Titular de la Facultad de Ingeniería de la Universidad Nacional de Chimborazo, Riobamba, Ecuador. E-mail: pvillacres@unach.edu.ec 
Al implementar este sistema de gestión de seguridad y salud en el trabajo se tendrá un mayor control e información de cada una de las áreas de trabajo en lo referente a seguridad y salud en el trabajo.

\subsection{Metodología}

La investigación es descriptiva, por el objetivo es aplicada, por el lugar es de campo ya que la investigación fue llevada a cabo en las oficinas administrativas ubicadas en Ibarra y en los diferentes proyectos que tiene la Empresa de Vialidad IMBAVIAL E.P. estos son: "Rehabilitación del Anillo Vial de Salcedo de 9,75 Km de longitud ubicado en la Provincia de Cotopaxi"; "Construcción de las Vías internas de la Cuidad del conocimiento YACHAY ubicado en la Provincia de Imbabura" y "Construcción de la Ampliación de las Instalaciones del Cuerpo de Bomberos de Otavalo, cerramiento del terreno adjunto a la Compañía de Bomberos de San Pablo del Lago y Construcción de las pistas de entrenamiento Bomberil". El método que se utilizó en la investigación es deductivo. Aplicativo en la legislación en seguridad y salud en el trabajo la Resolución 390 conocida como Reglamento General del Seguro de Riesgos de trabajo en el Art. 51 establece los parámetros del Sistema de Gestión (Jaramillo, 2011).

Al implementar este sistema se consideró cada una de los elementos y subelementos detallados en la Resolución 333 conocido como Sistema de Auditoria de Riesgos de Trabajo (Arias, 2009).

Se inició con la auditoria interna teniendo un porcentaje de cumplimiento de $0 \%$, elaborando en primer punto el Manual de Seguridad y Salud en el Trabajo, con cada uno de los procedimientos establecidos en el Artículo 8 de la Resolución 333. De ahí se procedió a revisar cada uno de los sub-elementos para poder generar los documentos que sirvieron de registros para poder verificar en una nueva auditoría interna de seguridad y salud en el trabajo, a la vez se utilizó otras normas aplicadas como son el Reglamento de Seguridad y Salud de los Trabajadores y mejoramiento del ambiente de trabajo Resolución 2393, Normas NEVI y el Regla- mento de Seguridad y Salud para la Construcción y Obras Públicas.

La identificación, medición y evaluación de los riesgos de trabajo se realizará mediante el uso de la Matriz de Evaluación de Riesgos Laborales realizada y aprobada por el Ministerio de Relaciones Laborales con la ayuda de la herramienta MS-Excel $\circledast$, para comprobar cada una de las hipótesis se utilizó el método del Chi cuadrado, con un antes y un después; se utiliza esta técnica debido a que se tienen resultandos cualitativos.

\subsection{Población y muestra}

La población se encuentra representada por todo el Personal que labora en la Empresa de Vialidad IMBAVIAL E.P. En total son 81 trabajadores, 66 pertenecen al género masculino y 14 al género femenino.

\section{RESULTADOS}

La valoración se realiza dividiendo la lista de comprobación en bloques de acuerdo a los elementos y subelementos involucrados del sistema de gestión los mismos que se asignaran los puntos que se indica en la Resolución 333, de esta manera se obtendrá una valoración global del cumplimiento de la norma por cada elemento involucrado. Para la presentación de resultados se muestran datos numéricos en porcentaje sobre el grado de cumplimiento de la norma y de cada elemento del Sistema de Gestión evaluado; para ello se utilizó el programa Excel, ver Tabla N. 1, se debe indicar que la empresa no tenía implementado la gestión de seguridad y salud en el trabajo antes del 01 de Julio del 2015.

\subsection{Resultado de la auditoria del sistema de ges- tión de seguridad y salud en el trabajo}

La auditoría del sistema de gestión de seguridad y salud en el trabajo se llevó a cabo el primero del julio por la Ing. Gabriela Uvidia en calidad de auditor interno. 
Tabla1. Resultados de la auditoria de riesgos de trabajo acorde a lo establecido en la Resolución C.D. 333.

\begin{tabular}{|c|c|c|c|c|c|c|c|c|c|c|}
\hline \multirow{2}{*}{\multicolumn{7}{|c|}{$\begin{array}{c}\text { AUDITORIA DEL SISTEMA DE GESTIÓN DE SEGURIDAD Y SALUD EN EL TRABAJO } \\
\text { UNIDAD DE SEGURIDAD Y SALUD EN EL TRABAJO }\end{array}$}} & \multicolumn{4}{|c|}{ FECHA:01/07/2015 } \\
\hline & & & & & & & & & & \multirow{4}{*}{ Respaldos } \\
\hline \multicolumn{2}{|c|}{ Requisito } & \multirow{3}{*}{ NORMATIVA } & \multirow{3}{*}{ Valor } & \multirow{3}{*}{$\begin{array}{c}\text { Cumple / } \\
\text { No } \\
\text { cumple }\end{array}$} & \multirow{3}{*}{ No Aplica } & \multirow{3}{*}{$\begin{array}{c}\text { Resultado } \\
\mathrm{A}\end{array}$} & \multirow{2}{*}{\multicolumn{2}{|c|}{$\begin{array}{c}\text { No } \\
\text { conformidad } \\
\end{array}$}} & \multirow{3}{*}{$\begin{array}{l}\text { Obser- } \\
\text { vación }\end{array}$} & \\
\hline \multirow{2}{*}{ Art. } & \multirow{2}{*}{ Inc } & & & & & & & & & \\
\hline & & & & & & & B & $\mathrm{C}$ & & \\
\hline 1 & & \multicolumn{9}{|c|}{ GESTIÓN ADMINISTRATIVA } \\
\hline \multicolumn{2}{|l|}{1.1} & POLITICA & & & & & & & & \\
\hline \multicolumn{2}{|l|}{ 1.1.a. } & $\begin{array}{l}\text { Corresponde a la naturaleza (tipo de actividad productiva) y magni- } \\
\text { tud de los factores de riesgos. }\end{array}$ & 0,125 & 1 & & & & & & \\
\hline \multicolumn{2}{|l|}{ 1.1.b. } & Compromete recursos. & 0,125 & 1 & & & & & & \\
\hline \multicolumn{2}{|l|}{ 1.1.c. } & $\begin{array}{l}\text { Incluye compromiso de cumplir con la legislación técnico legal de } \\
\text { seguridad y salud en el trabajo; y además, el compromiso de la em- } \\
\text { presa para dotar de las mejores condiciones de seguridad y salud } \\
\text { ocupacional para todo su personal. }\end{array}$ & 0,125 & 1 & & 1,00 & & & & \\
\hline \multicolumn{2}{|l|}{ 1.1.d. } & $\begin{array}{l}\text { Se ha dado a conocer a todos los trabajadores y se la expone en } \\
\text { lugares relevantes. }\end{array}$ & 0,125 & 1 & & & & & & \\
\hline \multicolumn{2}{|l|}{ 1.1.e. } & Está documentada, integrada-implantada y mantenida. & 0,125 & 1 & & & & & & \\
\hline \multicolumn{2}{|l|}{ 1.1.f. } & Está disponible para las partes interesadas. & 0,125 & 1 & & & & & & \\
\hline \multicolumn{2}{|l|}{$1.1 \mathrm{~g}}$. & Se compromete al mejoramiento continuo. & 0,125 & 1 & & & & & & \\
\hline \multicolumn{2}{|l|}{ 1.1.h. } & Se actualiza periódicamente. & 0,125 & 1 & & & & & & \\
\hline \multicolumn{2}{|l|}{1.2} & PLANIFICACIÓN & & & & & & & & \\
\hline \multicolumn{2}{|l|}{ 1.2.a. } & $\begin{array}{l}\text { Dispone la empresa de un diagnostico o evaluación de su sistema } \\
\text { de gestión, realizado en los dos últimos años si es que los cambios } \\
\text { internos así lo justifican. }\end{array}$ & 0,111 & & & & & & & \\
\hline $\begin{array}{l}0 \\
1.2 . \text { a. } 1\end{array}$ & & $\begin{array}{l}\text { Las no conformidades priorizadas y temporizadas respecto a la } \\
\text { gestión: administrativa; técnica; del talento humano; } y \text {, procedimien- } \\
\text { tos o programas operativos básicos. }\end{array}$ & 0,111 & 1 & & & & & & \\
\hline 1.2.b. & & $\begin{array}{l}\text { Existe una matriz para la planificación en la que se han temporizado } \\
\text { las No conformidades desde el punto de vista técnico. }\end{array}$ & 0,111 & 1 & & & & & & \\
\hline 1.2.c. & & La planificación incluye actividades rutinarias y no rutinarias; & 0,111 & 1 & & & & & & \\
\hline 1.2.d. & & $\begin{array}{l}\text { La planificación incluye a todas las personas que tienen acceso al } \\
\text { sitio de trabajo, incluyendo visitas, contratistas, entre otras; }\end{array}$ & 0,111 & 1 & & & & & & \\
\hline 1.2.e. & & $\begin{array}{l}\text { El plan incluye procedimientos mínimos para el cumplimiento de los } \\
\text { objetivos y acordes a las No conformidades priorizadas. }\end{array}$ & 0,111 & 1 & & & & & & \\
\hline 1.2.f. & & $\begin{array}{l}\text { El plan compromete los recursos humanos, económicos y tec- } \\
\text { nológicos suficientes para garantizar los resultados. }\end{array}$ & 0,111 & 1 & & 1,00 & & & & \\
\hline 1.2.g. & & $\begin{array}{l}\text { El plan define los estándares e índices de eficacia (cualitativa y/o } \\
\text { cuantitativa) del sistema de gestión de la seguridad y salud en el } \\
\text { trabajo, que permitan establecer las desviaciones programáticas, } \\
\text { en concordancia con el articulo } 11 \text { del presente reglamento. }\end{array}$ & 0,111 & 1 & & & & & & \\
\hline 1.2.h. & & $\begin{array}{l}\text { El plan define los cronogramas de actividades con responsables, } \\
\text { fechas de inicio y de finalización de la actividad. }\end{array}$ & 0,111 & 1 & & & & & & \\
\hline 1.2.i. & & El plan considera la gestión del cambio en lo relativo a: & 0,111 & & & & & & & \\
\hline $\begin{array}{l}0 \\
\text { 1.2. i.1. }\end{array}$ & & $\begin{array}{l}\text { Cambios internos.- Cambios en la composición de la plantilla, intro- } \\
\text { ducción de nuevos procesos, métodos de trabajo, estructura orga- } \\
\text { nizativa, o adquisiciones entre otros. }\end{array}$ & 0,056 & 1 & & & & & & \\
\hline $\begin{array}{l}0 \\
\text { 1.2. i.2. }\end{array}$ & & $\begin{array}{l}\text { Cambios externos. - Modificaciones en leyes y reglamentos, fu- } \\
\text { siones organizativas, evolución de los conocimientos en el campo } \\
\text { de la seguridad y salud en el trabajo, tecnologia, entre otros. Deben } \\
\text { adoptarse las medidas de prevención de riesgos adecuadas, antes } \\
\text { de introducir los cambios. }\end{array}$ & 0,056 & 1 & & & & & & \\
\hline 1.3. & & ORGANIZACIÓN & & & & & & & & \\
\hline 1.3.а. & & $\begin{array}{l}\text { Tiene Reglamento Interno de Seguridad y Salud en el Trabajo actu- } \\
\text { alizado y aprobado por el Ministerio de Relaciones Laborales; }\end{array}$ & 0,200 & 1 & & & & & & \\
\hline & & Ha conformado las unidades o estructuras preventivas: & 0,200 & & & & & & & \\
\hline & 1.3.b.1 & $\begin{array}{l}\text { Unidad de Seguridad y Salud en el Trabajo; dirigida por un profe- } \\
\text { sional con titulu de tercer o cuarto nivel, registrado en el SENECYT, } \\
\text { del área ambiental-biológica, relacionado a la actividad principal de } \\
\text { la empresa u organización, experto en disciplinas afines a los siste- } \\
\text { mas de gestión de la seguridad y salud ocupacional. }\end{array}$ & 0,050 & 1 & & & & & & \\
\hline 1.3.b. & 1.3.b.2 & $\begin{array}{l}\text { Servicio Médico de Empresa dirigido por un profesional con título } \\
\text { de Médico y grado académico de cuarto nivel en disciplinas afines } \\
\text { a la gestión de la seguridad y salud ocupacional, registrado por el } \\
\text { SENECYT; }\end{array}$ & 0,050 & 0 & & 095 & & & & \\
\hline & 1.3.b.3 & $\begin{array}{l}\text { Comité y Subcomités de Seguridad y Salud en el Trabajo, de con- } \\
\text { formidad con la ley. }\end{array}$ & 0,050 & 1 & & 0,95 & & & & \\
\hline & 1.3.b.4 & Delegado de seguridad y salud en el trabajo. & 0,050 & 1 & & & & & & \\
\hline 1.3.c. & & $\begin{array}{l}\text { Están definidas las responsabilidades integradas de seguridad } \\
\text { y salud en el trabajo, de los gerentes, jefes, supervisores, traba- } \\
\text { jadores, entre otros y las de especialización de los responsables de } \\
\text { las Unidades de Seguridad y Salud, y, Servicio Médico de Empresa, } \\
\text { asi como de las estructuras de SST. }\end{array}$ & 0,200 & 1 & & & & & & \\
\hline 1.3.d. & & $\begin{array}{l}\text { Están definidos los estándares de desempeño en seguridad y salud } \\
\text { en el trabajo. }\end{array}$ & 0,200 & 1 & & & & & & \\
\hline 1.3.e. & & $\begin{array}{l}\text { Existe la documentación del sistema de gestión de seguridad y } \\
\text { salud en el trabajo de la empresa u organización: manual, proced- } \\
\text { imientos, instrucciones y registros. }\end{array}$ & 0,200 & 1 & & & & & & \\
\hline
\end{tabular}




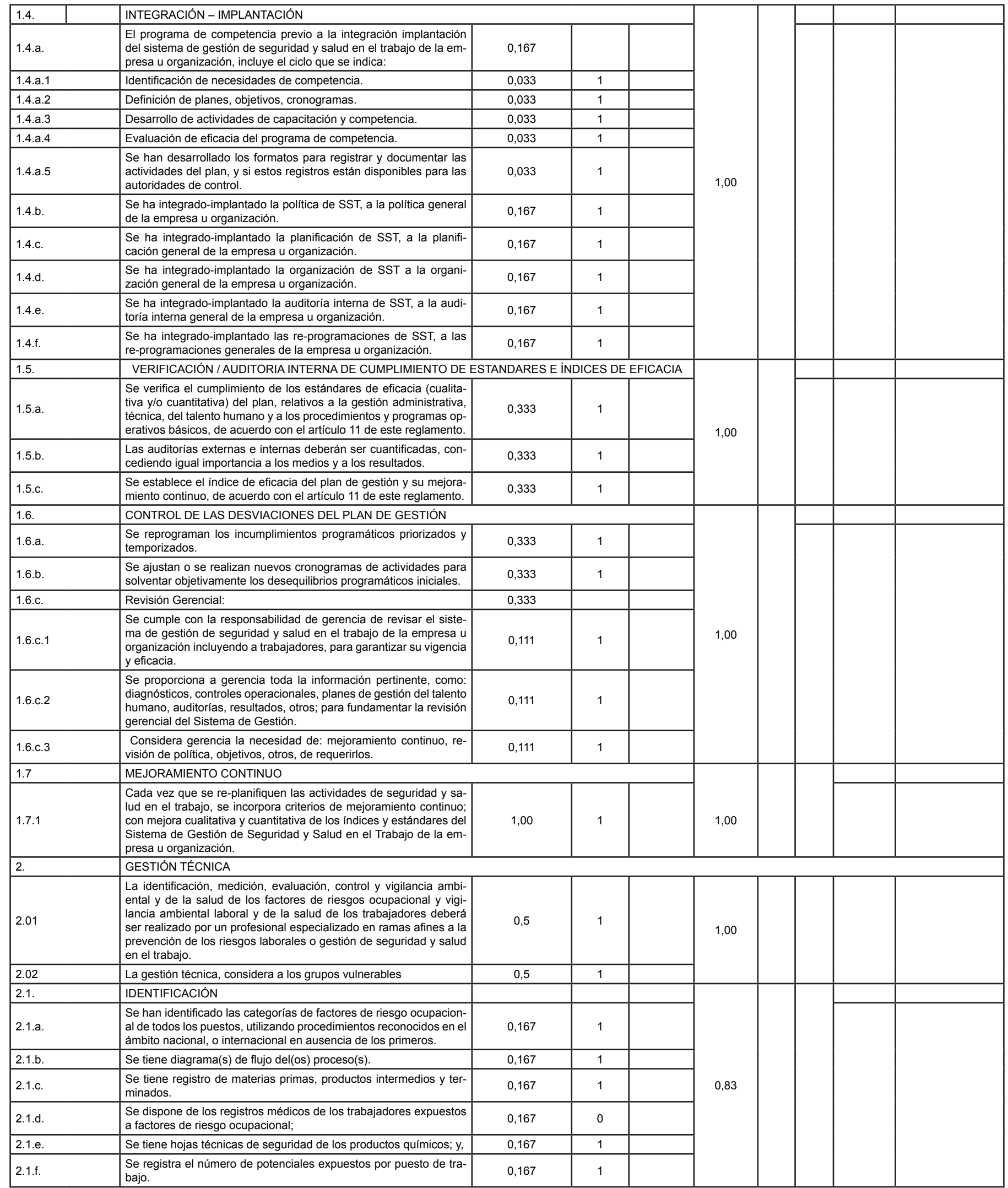




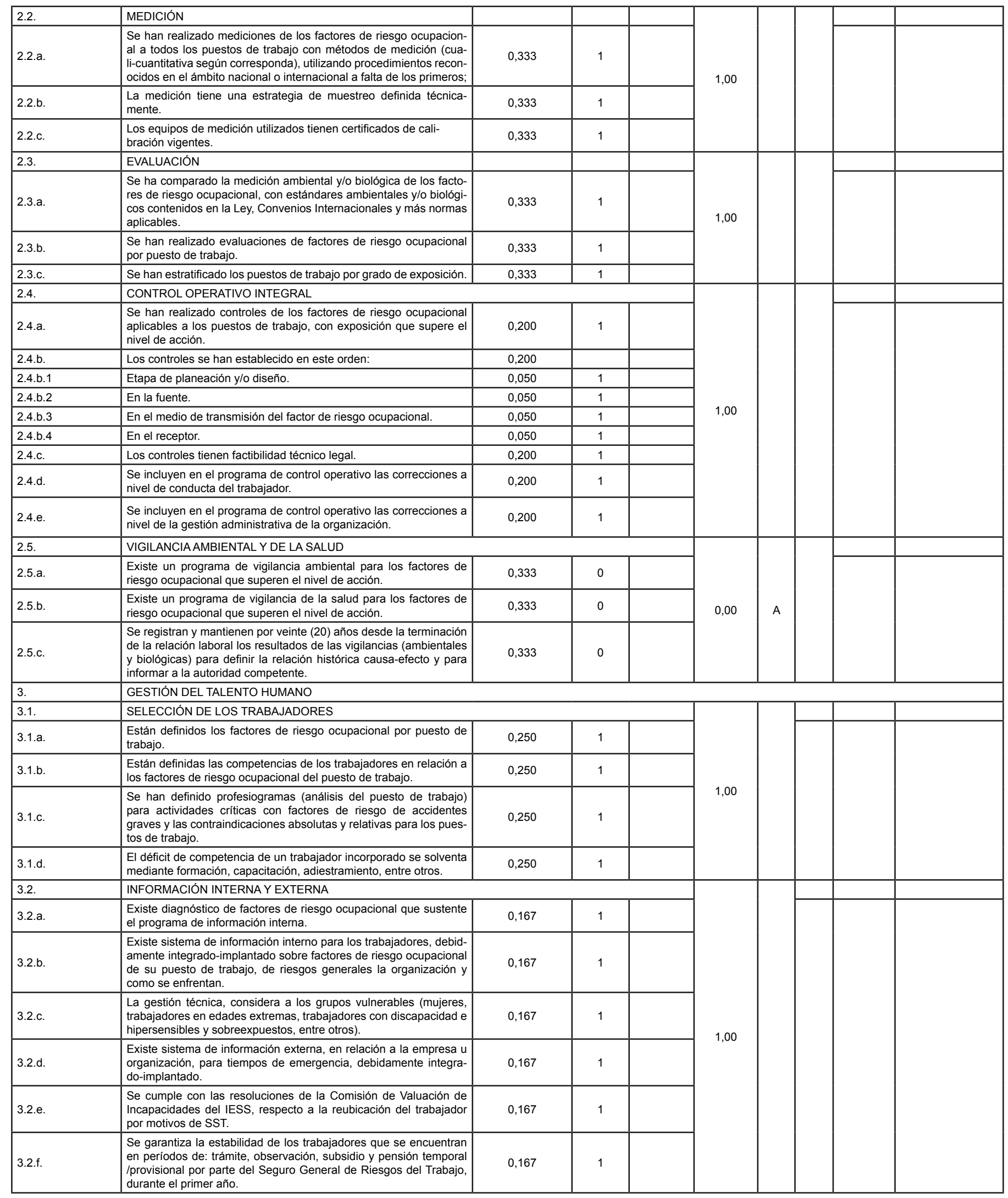




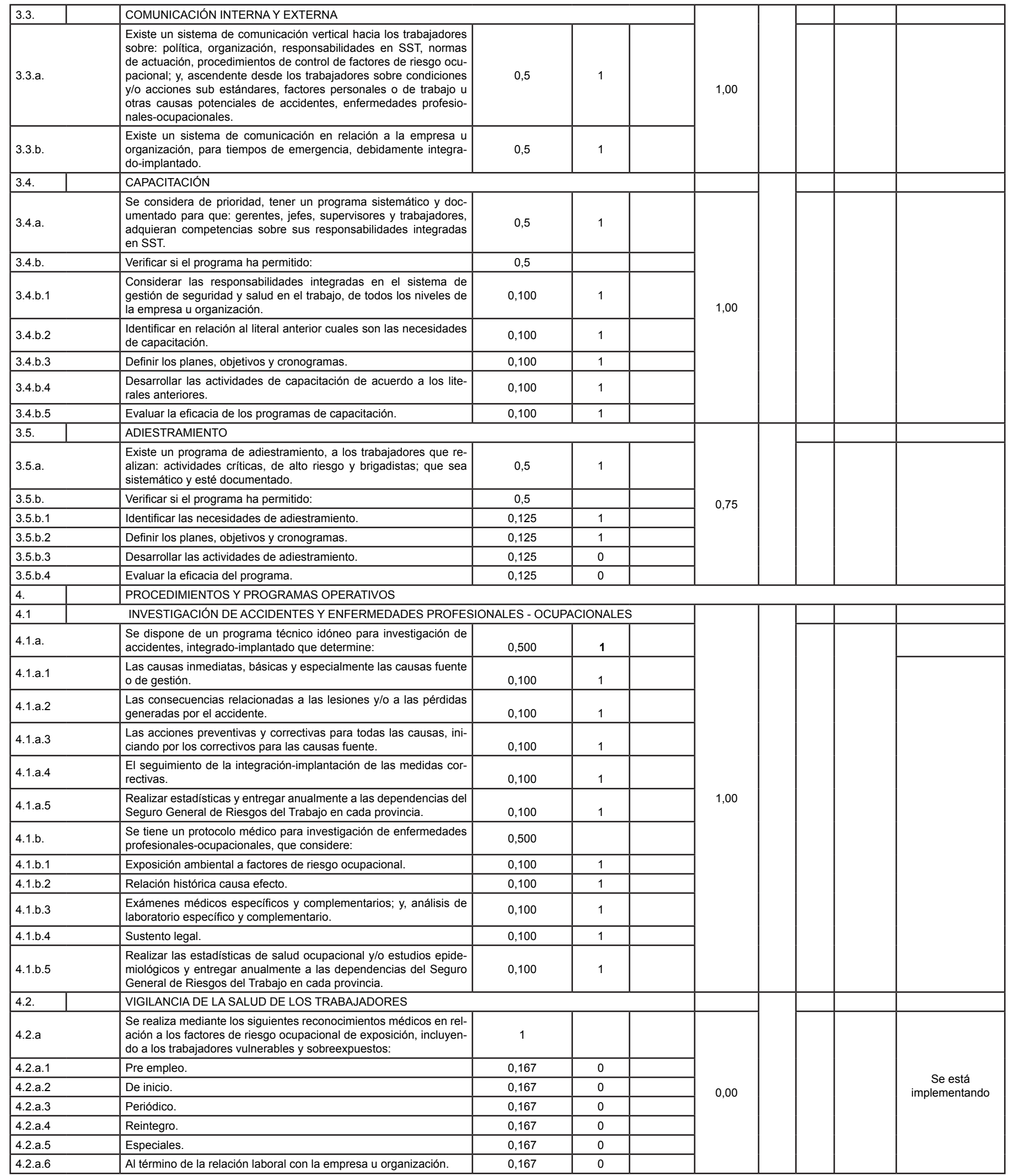




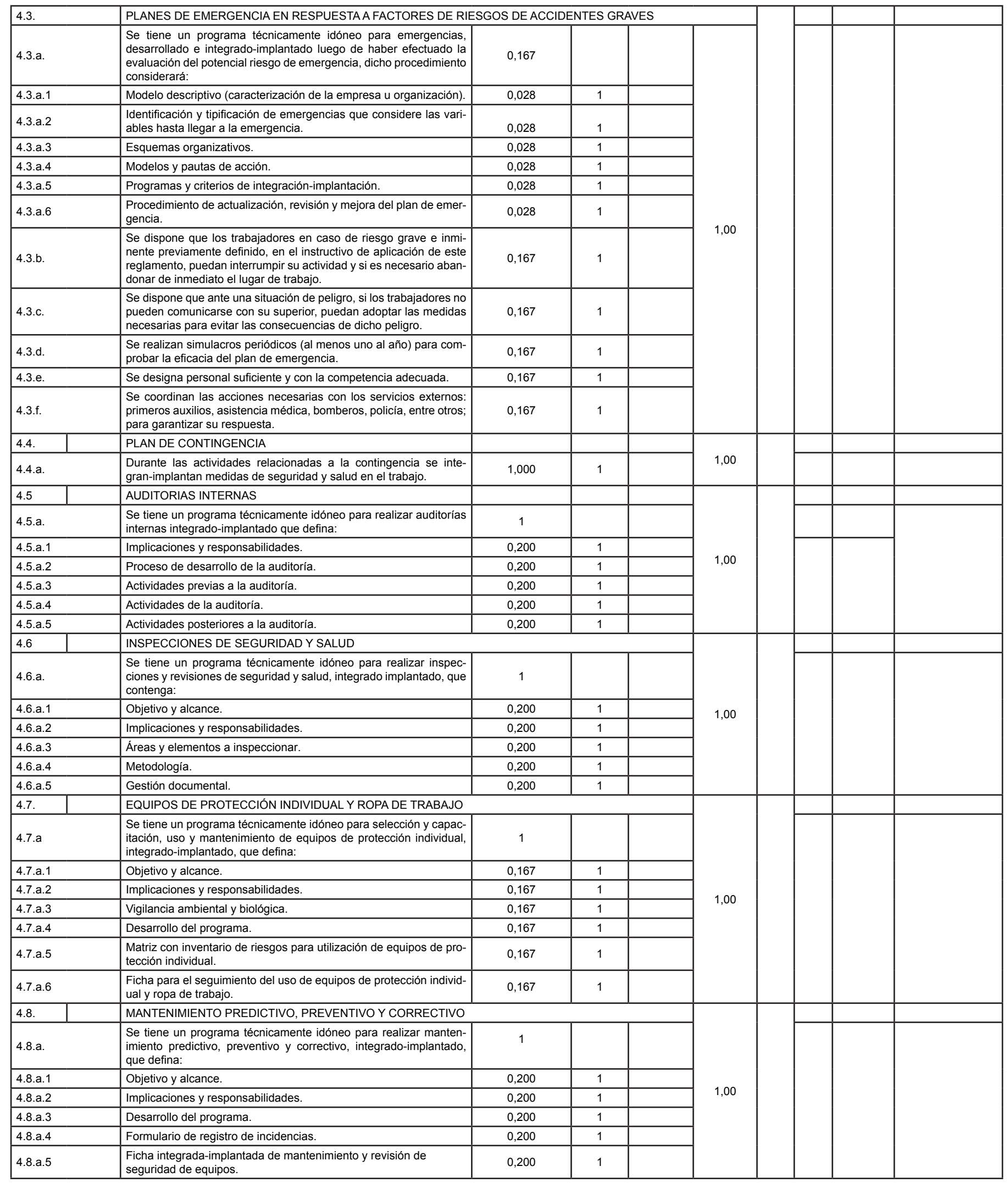

Fuente: Elaboración propia. 
En la Tabla N. 1 se evidencia la matriz en Excel esta fue elaborada con las cuantificaciones establecidas en la Resolución 333 SART "Sistema de Auditoria de Riesgos de Trabajo".

\section{DISCUSIÓN RESULTADOS}

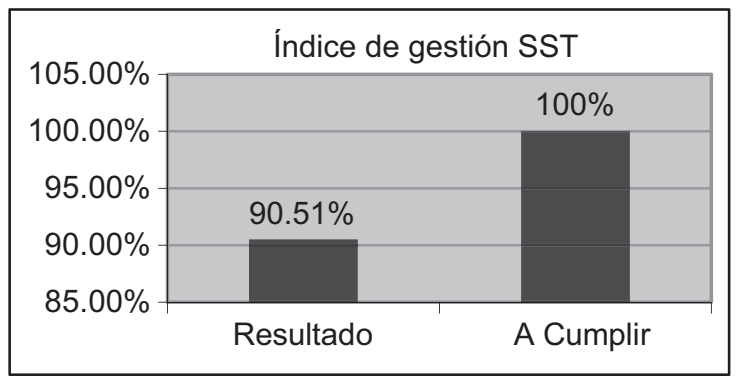

Fuente: Elaboración propia.

Figura 1. Cumplimiento del SART en la Empresa de Vialidad IMBAVIAL E.P.

De acuerdo a la Figura 1, la Empresa de Vialidad IMBAVIAL E.P. tiene un cumplimiento del $90.51 \%$ del Sistema de Gestión de Seguridad y Salud en el Trabajo. Mejoro el ambiente de trabajo, se creó una conciencia ambiental, la empresa no generó multas considerando el uno por mil por día de retraso en el monto del proyecto. Teniendo en cuenta la legislación vigente a nivel nacional la Empresa de Vialidad IMBAVIAL E.P., mantiene su sistema de gestión y seguridad en el trabajo sobre el $80 \%$ de cumplimiento. La Empresa se encuentra preparada ante una auditoria de riesgos de trabajo. Para el año 2016 se aprobado un presupuesto de cien mil dólares para el área de seguridad y salud en el trabajo debido a que los trabajadores demostraron ser más productivos en cada uno de los puestos de trabajo al implementar el Sistema de Gestión de Seguridad y Salud en el Trabajo.

\subsection{Comprobación de hipótesis}

El Sistema de Gestión de Seguridad y Salud para la Empresa de Vialidad IMBAVIAL E.P. en la provincia de Imbabura, genera un ambiente de trabajo seguro en el periodo enero - julio 2015, con la implementación de la política, organización, planificación, implementación, evaluación y seguimiento de los planes.

Para la comprobación de la hipótesis se realizó una encuesta con veinte preguntas, ver Tabla 2 esta encuesta fue validada por la Gerencia General. Esta encuesta se aplicó a los 81 trabajadores que laboran en la Empresa de Vialidad IMBAVIAL E.P.

Los resultados fueron tabulados en una hoja de Excel, luego se procedió aplicar el chi cuadro para comprobar si la hipótesis es afirmativa o nula.

Tabla 2. Comprobación de hipótesis general

\begin{tabular}{|c|c|c|c|c|c|c|}
\hline \multirow{2}{*}{ N. ${ }^{\circ}$} & \multirow{2}{*}{ PREGUNTAS } & \multirow{2}{*}{ sí } & \multirow{2}{*}{ NO } & \multirow{2}{*}{ SUB-TOTAL } & \multicolumn{2}{|c|}{ TABLA ESPERADA } \\
\hline & & & & & sí & $\mathrm{NO}$ \\
\hline 1 & $\begin{array}{l}\text { Al implementar el Sistema de Gestión de Seguridad y Salud en el Trabajo en la } \\
\text { Empresa, la empresa brindo un ambiente laboral seguro. }\end{array}$ & 72 & 9 & 81 & 4 & 0 \\
\hline 2 & $\begin{array}{l}\text { Al implementar la política de seguridad y salud en el trabajo por la Gerencia Gene- } \\
\text { ral, se garantiza un ambiente de trabajo adecuado. }\end{array}$ & 73 & 8 & 81 & 4 & 0 \\
\hline 3 & $\begin{array}{l}\text { Al difundir la planificación y colocar en lugares de relevantes el trabajador controla } \\
\text { las actividades planificadas, se está garantizando que el trabajador desarrolle sus } \\
\text { actividades en un ambiente adecuado de trabajo. }\end{array}$ & 72 & 9 & 81 & 4 & 0 \\
\hline 4 & $\begin{array}{l}\text { Al implementar la Unidad de Seguridad, Salud en el Trabajo y ambiente, se está } \\
\text { brindando un mejor ambiente de trabajo. }\end{array}$ & 70 & 11 & 81 & 4 & 1 \\
\hline 5 & $\begin{array}{l}\text { Al aprobar recursos por la Gerencia General para la Unidad de Seguridad, Salud } \\
\text { en el Trabajo y Ambiente se está garantizando un ambiente de trabajo seguro }\end{array}$ & 68 & 13 & 81 & 3 & 1 \\
\hline 6 & $\begin{array}{l}\text { Al identificar los peligros y evaluar los riesgos se está garantizando un ambiente } \\
\text { de trabajo seguro. }\end{array}$ & 70 & 11 & 81 & 4 & 1 \\
\hline 7 & $\begin{array}{l}\text { Al realizar las mediciones de ruido se pudo verificar que estaban dentro de los } \\
\text { límites permisibles que establece la Norma } 2393 \text {. Esta medición garantizo que el } \\
\text { trabajador desarrolla sus actividades en un ambiente seguro de trabajo. }\end{array}$ & 68 & 13 & 81 & 3 & 1 \\
\hline 8 & $\begin{array}{l}\text { Al implementar las pausas activas en la empresa, aprobado por la Gerencia Gene- } \\
\text { ral con el objetivo de controlar los niveles de estrés de los trabajadores; garantizó } \\
\text { un ambiente seguro de trabajo }\end{array}$ & 66 & 15 & 81 & 3 & 1 \\
\hline 9 & Al ejecutar las pausas activas se disminuyó los dolores lumbares y articulaciones. & 72 & 9 & 81 & 4 & 0 \\
\hline 10 & Al evaluar el riesgo ergonómico se garantizó un adecuado ambiente de trabajo. & 72 & 9 & 81 & 4 & 0 \\
\hline 11 & $\begin{array}{l}\text { Conoce usted de los riesgos laborales de su puesto de trabajo las medidas de } \\
\text { prevención. }\end{array}$ & 72 & 9 & 81 & 4 & 0 \\
\hline
\end{tabular}




\begin{tabular}{|c|l|c|c|c|c|c|}
\hline 12 & Su área de trabajo está bien organizada & 71 & 10 & 81 & 4 \\
\hline 13 & Las condiciones de trabajo de su puesto de trabajo son seguras & 73 & 8 & 81 & 4 & 0 \\
\hline 14 & Las cargas de trabajo están bien repartidas & 73 & 8 & 81 & 4 & 0 \\
\hline 15 & Mantiene el orden y la limpieza de su puesto de trabajo & 72 & 9 & 81 & 4 & 0 \\
\hline 16 & El trabajo que usted realiza. Lo ejecuta de forma segura & 73 & 8 & 81 & 4 & 0 \\
\hline 17 & $\begin{array}{l}\text { Los protocolos de emergencia son claros. Sabe usted cómo actuar en alguna } \\
\text { emergencia }\end{array}$ & 74 & 7 & 81 & 4 & 0 \\
\hline 18 & $\begin{array}{l}\text { La empresa le facilita los EPP y Ropa de trabajo acorde los riesgos a los que se } \\
\text { encuentran expuestos. }\end{array}$ & 73 & 8 & 81 & 4 & 0 \\
\hline 19 & $\begin{array}{l}\text { Al ejecutar las inspecciones de seguridad y salud en el trabajo se garantiza que el } \\
\text { trabajador desarrolle sus actividades en un ambiente seguro de trabajo }\end{array}$ & 74 & 7 & 81 & 4 & 0 \\
\hline 20 & $\begin{array}{l}\text { Al ejecutar el mantenimiento periódico a cada una de las maquinarias se garantiza } \\
\text { que el trabajo ejecutado por usted se realiza en un ambiente seguro de trabajo }\end{array}$ & 72 & 9 & 81 & & 4 \\
\hline & SUBTOTAL & 1430 & 190 & 1620 & Chi cuadrado calculado & 32,133 \\
\hline & & & & & Chi cuadrado tabulado & 31,659 \\
\hline
\end{tabular}

Fuente: Elaboración propia.

Luego se procedió a graficar en la curva de Gauss, donde se obtuvo la siguiente curva:

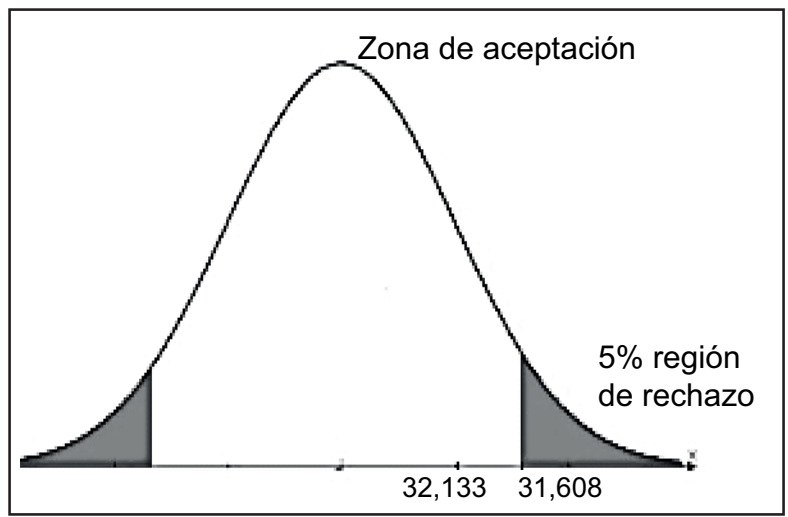

Fuente: Elaboración propia.

Figura 2. Curva de Gauss.

En la Figura 2 se observa que 32,133 es mayor a 31,658 por lo tanto se demuestra que la hipótesis es positiva.

El Sistema de Gestión de Seguridad y Salud para la Empresa de Vialidad IMBAVIAL E.P. en la provincia de Imbabura, genera un ambiente de trabajo seguro en el periodo enero - julio 2015 ,

\section{CONCLUSIONES}

- La Implementación del Sistema de Gestión de Seguridad y Salud en el Trabajo, para la Empresa de Vialidad IMBAVIAL E.P. en la provincia de Imbabura, genera un ambiente de trabajo seguro en el periodo enero - julio 2015; considerando la anterior auditoria interna de riesgos de trabajo la empresa se encontraba con $0 \%$ del cumplimiento de la legislación vigente en seguridad y salud en el trabajo, actualmente se encuentra con más del $80 \%$.

- El Sistema de Gestión de Seguridad y Salud mediante la Gestión Administrativa para la Empresa de Vialidad IMBAVIAL E.P. en la provincia de Imbabura, genera un ambiente de trabajo seguro en el periodo enero - julio 2015; Actualmente la gerencia recepta informes mensuales de la implementación del sistema de gestión teniendo conocimiento en cada uno de los elementos y subelementos.

- El Sistema de Gestión de Seguridad y Salud mediante la Gestión Técnica para la Empresa de Vialidad IMBAVIAL E.P. en la provincia de Imbabura, genera un ambiente de trabajo seguro en el periodo enero - julio 2015; Ahora el personal tiene control periódico y gestión de los riesgos presentes minimizando el impacto negativo que tenía el personal.

- El Sistema de Gestión de Seguridad y Salud mediante la Gestión de Talento Humano para la Empresa de Vialidad IMBAVIAL E.P. en la provincia de Imbabura genera un ambiente de trabajo seguro en el periodo enero - julio 2015; Ahora se tiene personal calificado y bien seleccionado que permite que se realicen los trabajos con eficiencia y eficacia, además se tiene cronograma establecido para mejorar las competencias de cada uno de los trabajadores.

- El Sistema de Gestión de Seguridad y Salud mediante los Procesos Operativos básicos, para la Empresa de Vialidad IMBAVIAL E.P. en la provincia de Imbabura, genera un ambiente 
de trabajo seguro en el periodo enero - julio 2015; Ahora con esta implementación del sistema de gestión el personal tiene al día sus herramientas, maquinarias y/o equipos, tienen su ropa de trabajo, equipo de protección personal acorde a los riesgos de trabajo a los cuales se encuentran expuestos, se actualiza constantemente los índices reactivos y proactivos, reportándose de manera semestral al Instituto Ecuatoriano de Seguridad Social departamento de Riesgos Laborales.

\section{RECOMENDACIONES}

- Mantener el sistema de gestión de seguridad y salud en el trabajo con un técnico especialista en materia de seguridad y salud en el trabajo.

- Mantener los informes generales hacia la gerencia general acerca del sistema de gestión de seguridad y salud en el trabajo.

- Realizar de manera periódica la evaluación de factores de riesgos en cada uno de los frentes de trabajo.
- Implementar un sistema de Gestión de Seguridad y Salud en el Trabajo y certificar a la Empresa.

\section{REFERENCIAS BIBLIOGRÁFICAS}

[1] Arias, D. P. (2009). Sistema de Auditoría de Riesgos de Trabajo. Quito: IESS.

[2] Jaramillo R. (2011). Resoluciòn C.D. Nª 390. Quito.

[3] Luis Vazques. (31 de diciembre de 2014). Datum Corporaticon. Obtenido de Web Site: https://www.iess.gob.ec/documents/10162/3780 216/2015+04+01+Rendicion+de+cuentas+v3. pdf

[4] Torres, S. A. (01 de MAYO de 2015). 42 de cada 1000 trabajadores sufren accidentes laborales en nuestro país. El Comercio, pág. 1. 